\title{
Au-delà des limites : pour un nouvel imaginaire culturel
}

Beyond Limits: Towards a new cultural imaginary

Gregory B. Lee

\section{(2) OpenEdition}

Journals

Édition électronique

URL : http://journals.openedition.org/transtexts/1223

DOI : $10.4000 /$ transtexts. 1223

ISSN : 2105-2549

Éditeur

Gregory B. Lee

Référence électronique

Gregory B. Lee, "Au-delà des limites : pour un nouvel imaginaire culturel », Transtext(e)s Transcultures 跨文本跨文化 [En ligne], 13 | 2018, mis en ligne le 01 décembre 2018, consulté le 03 juillet 2019. URL http://journals.openedition.org/transtexts/1223; DOI : 10.4000/transtexts.1223

Ce document a été généré automatiquement le 3 juillet 2019

(c) Tous droits réservés 


\title{
Au-delà des limites : pour un nouvel imaginaire culturel
}

\author{
Beyond Limits: Towards a new cultural imaginary
}

Gregory B. Lee

1 Je voudrais discuter des limites, des conséquences de leur absence, dans ce que nous appelons l'ère anthropocène, et de la nécessité d'en avoir pour pouvoir envisager l'avenir pour du monde. Par «le monde»j'entends cette condition qui existe et qui a été reconnue par les philosophes il y a plus de 2000 ans. C'est-à-dire ce que nous avons appelé depuis la nature, mais une nature qui intègre l'humain, la physis avant la séparation, ce que les philosophes taoïstes à l'époque appelaient les «myriades de choses », l'univers. Ils appelaient à renverser, à revenir sur cette séparation qui déjà à travers l'agriculture, la ville, les grands travaux publics avait l'ambition de dominer, et en partie avait déjà dominé, la « nature ».

2 Mais une stratégie négative de limites suffit-elle? Et peut-elle convaincre et inspirer les jeunes générations et les générations futures à imaginer et à refaire le monde ? Il faudrait peut-être aussi installer un nouvel imaginaire basé sur la créativité et l'épanouissement de chacune et chacun. Mais, tournons-nous d'abord vers la nécessité des limites.

3 Jacques Ellul et Bernard Charbonneau, avaient déjà repéré le problème posé par les limites, et les dégâts provoqués par l'illimité en 1935. Ils avaient spécifiquement identifié la «technique » comme «fait décisif de la modernité »; ici j'emprunte le résumé de Quentin Hardy :

[L]e fait décisif de la modernité: la technique fait triompher la raison instrumentale. La technique est beaucoup plus qu'un ensemble de procédés industriels, c'est un 'procédé général', c'est à dire un imaginaire de pratiques tournées vers la production illimitée, l'efficacité et la multiplication de dispositifs abstraits et opprimants. Plongé dans un milieu qui n'est que moyens, intégralement médiatisé, et donc sans prise sur son environnement, l'homo technologicus, se retrouve dans un univers de fatalités où toutes les grandeurs (économie, médias, industrie, travail) le dépassent. ${ }^{1}$ 
clairement et visiblement incarnée dans les premières formes du "capitalisme", programmatiquement exprimée dans les philosophes rationalistes du XVII siècle (Descartes, Leibniz)» ce qui donnera «le capitalisme industriel...puis l'invasion de la 'rationalisation' dans tous les domaines de l'activité sociale, et finalement la course folle de la techno-science autonomisée que nous connaissons aujourd'hui ». ${ }^{2}$

5 Une, sinon la principale, ambition de Charbonneau et Ellul fut de mettre en question de façon radicale la présomption que la technique est neutre (par technique je veux dire non seulement ce que nous appelons « la technologie », mais aussi les procédés techniques) :

[La technique...] engendre le développement de la puissance. Loin de permettre l'émancipation, la puissance engendre des déséquilibres et devient incontrôlable, car elle appelle nécessairement la concentration et le gigantisme. ${ }^{3}$

Plus tard, en 1977, Ellul identifiera l'inverse dans les relations entre l'humain et les innovations, quand il constate :

[...U]n groupe humain cherche à " persévérer dans l'être », n'adopte les innovations que progressivement et tend à les absorber : or nous avons vu que la relation s'est inversée : maintenant c'est la technique qui englobe et qui détermine les formes culturelles, la « civilisation». Mais ceci n'est ni accepté ni achevé. ${ }^{4}$

Quand Ellul discute des limites, il s'appuie sur Ivan Illich et fait ressortir la distinction entre seuils et limites :

Les seuils représentent les bornes entre lesquelles l'action de l'homme (et la technique) doit se situer pour que la survie reste possible. Il s'agit de nécessités. Et lorsque nous parlons de nuisances, de pollution, d'épuisement des ressources, nous désignons des seuils.... Il s'agit alors simplement des conditions de survie. Mais ceci n'est rien pour la création d'une civilisation, d'une culture: ici l'homme doit se fixer à lui-même des limites qui constituent le dessin d'une culture. Ici parait le volontaire et le délibératif... la "croissance zéro" n'est en rien la garantie d'apparition d'une culture nouvelle, seulement la possibilité.... C'est la fixation des limites qui est créatrice de liberté, contrairement à ce que l'on croit..., je ne crois que rien n'est aussi fondamental que ce problème des limites volontaires. ${ }^{5}$

Pour Castoriadis, ce ne sont que ces limites volontaires qui sont valables:

La seule limitation véritable que peut comporter la démocratie est l'auto-limitation, qui ne peut être, en dernière analyse, que la tâche et l'œuvre des individus (des citoyens) éduqués par et pour la démocratie.... Autant dire que la démocratie, comme la philosophie, écarte nécessairement le sacré...[qu']elle exige que les êtres humains acceptent dans leur comportement réel ce qu'ils n'ont presque jamais voulu accepter vraiment.... à savoir qu'ils sont mortels. ${ }^{6}$

Plus tard, en 1990, Castoriadis reparle des limites et de la «cohabitation» de l'humanité avec la nature. Et ici on entend des échos de quelques philosophes éclairés, que nous appelons aujourd'hui grecs et chinois, qui pensaient qu'étant donnée la séparation irréparable, la rupture qu'était l'auto-soustraction de l'humanité du monde, de ses rythmes et de ses pratiques, dont elle avait jusqu'alors fait partie, le maximum que nous pouvions imaginer était la convergence, ce que les Taoïstes préconisaient dans la formule tian ren he yi 天人合一, le monde naturel et l'humanité harmonieusement unis. Cette formule reconnaissait que la condition originaire et idéale de l'humanité en tant que partie intégrante de ce monde était bel et bien perdue, et que la séparation et l'aliénation s'étaient depuis longtemps installées.

Pour Castoriadis, et dans des termes qui nous rappellent Ellul et Illich : 
Un changement d'attitude envers la nature est indispensable. Nous devons nous défaire des fantasmes de la maîtrise et de l'expansion illimitées, arrêter l'exploitation sans bornes de notre planète, cohabiter avec elle amoureusement, comme un jardinier anglais. ${ }^{7}$

11 Sauf que, comme nous le savons, le jardin anglais est très léché, très maitrisé et a longuement été bourré de pesticides... mais passons. Castoriadis vise ici, dans son jardin idéalisé, une "cohabitation» avec une "nature» qui serait toujours séparée de l'humanité. Peut-être que, comme dans la Cité grecque qui lui était si chère, il s'agit d'une vision pragmatique dans lequel le jardin anglais serait déjà préférable au parking bétonné. Mais d'une façon semblable à celle d'André Gorz, qui lui non plus n'a jamais abandonné la téléologie judéo-chrétienne-marxiste, Castoriadis ne conçoit pas le monde en tant que monade, ou unité maximale, à laquelle l'homme appartiendrait. Néanmoins, Castoriadis va aussi loin qu'il le peut étant données les contraintes de sa vision grécooccidentaliste des choses lorsqu'il écrit :

L'autonomie, la liberté, n'est pas seulement l'abolition des contraintes externes ou des pulsions psychiques; elle est aussi l'établissement d'un autre type de rapport entre nos poussées profondes, individuelles ou collectives, et des instances capables d'en faire le tri, de leur donner forme ou de les empêcher de se manifester dans la réalité. C'est le rôle de la subjectivité réfléchissante et délibérante au plan individuel, des institutions démocratiques au plan collectif car la démocratie est le régime de la réflexivité collective et de la liberté autolimitée. ${ }^{8}$

C'est ici qu'il conviendrait de revenir à la philosophie Taoïste que j'ai évoquée auparavant, car nous venons d'évoquer deux éléments clés, le pouvoir et le vouloir.

La Chine, par Chine, j'entends la République populaire de Chine établie en 1949, par Chine, je veux dire aussi cette idée d'un état-nation "Chine ", imaginée à partir de la fin du dix-neuvième siècle pour être construite pendant les vingtième et vingt-et-unième. Cette Chine-là n'est point celle que les Européens nommèrent la «Chine » à partir du seizième siècle, cette Chine que nous appelons parfois « la Chine ancienne ».

Cependant, cette Chine n'existait pas, ni dans la réalité ni dans l'imagination, au temps des philosophes que nous appelons les Taoïstes ou daojia 道家, l'école taoïste, ceux qui concevaient le Dao 道, la Voie, comme un mode de vie. Il y a entre 2000 et 2500 ans, ceux qui nous laissèrent leurs textes philosophiques habitaient des états disparates et pratiquaient une langue totalement distincte de la langue chinoise qu'on utilise dans la Chine de Xi Jinping, au moins aussi distincte que le français d'aujourd'hui ne l'est du latin de Cicéron. ${ }^{9}$

15 Le Taoïsme dont je parle ne relève pas du mysticisme oriental, mais plutôt d'une réflexion fondée sur l'observation que, de nos jours, nous qualifierions de «scientifique "; il ne s'agit pas de croyance, ni de religions - je laisse de côté la religion populaire taoïste qui a émergé par la suite - mais de philosophie. Il ne s'agit pas non plus de "sagesse ", mais d'une réflexion souvent politique et toujours philosophique. On ne parle pas non plus d'une démission anarcho-conservatrice face au pouvoir, mais d'une réflexion critique des pratiques politiques.

16 Le monde des Taoïstes était également celui de Confucius, qui élaborait, lui, une philosophie, une idéologie du pouvoir pratique, de gouvernance, qui privilégiait l'urbain et les grands travaux. ${ }^{10}$ Ellul avance une thèse très intéressante selon laquelle l'histoire narrée par la Bible est également l'histoire de la marche de la campagne, du sauvage, vers la ville, la civilisation. Tout commence dans le jardin d'Eden pour finir dans l'utopie de 
Jérusalem. Motif repris par l'écrivain William Blake dans son poème de 1804 « And did those feet in ancient time » dont la dernière strophe nous annonce :

I will not cease from Mental Fight,

Nor shall my Sword sleep in my hand :

Till we have built Jerusalem,

In England's green \& pleasant Land. ${ }^{11}$

17 Le paradis est dans la ville qu'on construit à partir de la «green and pleasant land » et contre celle-ci.

La philosophie de Confucius suit une logique semblable, l'environnement autour de l'homme, pour les Confucéens, est là pour être maîtrisé et exploité pour construire le monde parfait, ou plutôt pour Confucius pour reconstruire car ce monde, cette entité globale gouvernée par un souverain suprême, qui aurait déjà existé dans le passé lointain. Ce monde est construit autour d'une civilisation urbaine, contenue derrière des murs, éloignée de la campagne et des paysans. Pour Confucius, ce qui compte c'est le monde politique, la gouvernance, les règles de surface, les rites, c'est-à-dire le vernis, l'urbanité.

Pour les Taoïstes tels Zhuangzi 薪子, Laozi 老子 et Liezi 列子, cette conception du monde de Confucius constitue le degré zéro de la vie humaine. Et pour Zhuangzi, le monde qu'il faut retrouver n'est pas un monde de rois-sages mythiques, mais un moment d'avant la civilisation humaine, avant la dominance de l'urbain, avant notre séparation du monde que nous aurions extériorisé.

20 Comme nous l'a expliqué si brillamment notre collègue Jean François Billeter, l'idée reçue que Zhuangzi, ou au moins les textes qu'on lui attribue, «évite de se commettre avec le pouvoir ", est fausse. Mais c'est une «idée de tous les commentaires chinois, qu'ont reprise les sinologues occidentaux. » Comme le démontre Billeter, " plus qu'un rejet, il y a chez Zhuangzi une critique radicale du pouvoir. $»^{12}$ C'était un des commentaires de ses textes, un des manipulateurs idéologiques de l'époque, qui a réduit le Taoïsme, et en particulier Zhuangzi, à une peau de chagrin dans la panoplie syncrétique de l'idéologie qui servait à maintenir le pouvoir monarchique après l'unification des états en ce que nous appelons à présent «l'empire » ou « la Chine impériale ». C'est un dénommé Guo Xiang 郭象 qui «a transformé une critique acérée du pouvoir en une apologie de la démission et de l'indifférence morale. ${ }^{13}$ C'est en émasculant Tchouang-tseu [Zhuangzi] qu'il lui a assuré une place durable dans la culture des classes dominantes $»{ }^{14}$

21 L'analyse de Zhuangzi sur le vouloir est que « les hommes usent leurs forces et courent à leur perte parce qu'ils sont les jouets de leur propre vouloir ", un constat qui nous permet de réfléchir de nouveau aux limites volontaires. ${ }^{15}$ Dans la pensée que nous retrouvons dans le texte de Zhuangzi le « vouloir » et «le pouvoir » sont imbriqués. Le pouvoir dont parle le Zhuangzi est le pouvoir moral, le pouvoir de maîtriser le vouloir. Le Zhuangzi nous dit que « la conscience peut [...] acquérir le pouvoir [...] de vouloir ou de ne pas vouloir, et se libérer par là de l'erreur $» .^{16}$

22 Jacques Ellul, lui, parle de la «non-puissance ", non pas de l'impuissance, mais plutôt d'une décision consciente de ne pas utiliser notre pouvoir, de nous limiter, comme jusqu'ici ceux qui nous gouvernent ont réussi à le faire avec les armes nucléaires. Zhuangzi a également parlé du non-exercice du pouvoir qui passe par la maitrise de la volonté, du vouloir.

23 L'Ellul de mi-carrière, l'Ellul de 1966, évoqua ce que Castoriadis allait qualifier d'imaginaire, Ellul nous appelle à réfléchir aux lieux communs, dans son livre Exégèse de 
nouveaux lieux communs, qui rappelle Exégèse de lieux communs de Léon Bloy rédigé au début du vingtième siècle et qui critiquait la société bourgeoise. Les lieux communs, tels que « on ne peut pas arrêter le progrès » sont la clé de voûte de cet imaginaire, voire de cette idéologie, qui nous rend si obéissants face à la machine destructrice dont l'anthropocène est le produit.

[...ce] catalogue des illusions collectives, représentations inconsciemment faussées des autres...aussi bien qu'exaltation inconsciemment valorisée des idéaux que l'on prétend avoir... croyances collectives, reposant sur des présuppositions admirées sans discussion, sans contestation possibles. Le lieu commun est vraiment commun parce qu'il ne supporte aucune discussion de base. Il sert à tous comme pierre de touche, comme instrument de reconnaissance. ${ }^{17}$

Mais les coupables ne se limitent pas de nos jours à l'ogre, à l'homme fort, à une classe dirigeante, à un pays méchant, comme dit Ellul, « tous les hommes sont engagés dans un processus d'évolution commun [...] Notre monde est devenu total dans ses œuvres et ses expressions, et cette unité dépasse de loin toutes les scissions fussent-elles aussi graves que la classe ou la nation $»{ }^{18}$

Comme je l'ai décrit ailleurs, la Chine que l'on montre du doigt lorsque nous parlons du changement climatique, de la dégradation de la planète ou de l'épuisement des ressources naturelles n'est pas plus responsable que les autres. La Chine est peut-être à présent chargée de tourner la manivelle de l'orgue de barbarie qu'est la techno-économie mondiale, mais elle seule ne peut pas cesser de tourner, ne peut pas changer la musique de la machine. ${ }^{19}$ Cela ne peut être fait que par l'exercice d'une volonté de fixer des limites. Et si Castoriadis a raison, et si la limitation valable est l'auto-limitation, il nous reste du chemin à faire, s'il nous reste seulement le temps de le parcourir.

Dans son livre récent Demain l'Europe, Billeter expose la vision pour une nouvelle Europe qui serait une république que les Européens institueront «s'ils y voient le moyen de satisfaire leurs besoins et leurs désirs essentiels ou de moins œuvrer en faveur d'une société qui les satisfera. $»^{20}$

Mais, quelle stratégie poursuivre pour convaincre une majorité hégémonique d'adopter de telles mesures? Et qui, quel sujet ou quel groupe de sujets, quelle élite mènera ce combat? Comment établir un projet concret mondial, ou plus modestement et simplement européen, sans avoir non seulement le consentement mais aussi la participation du plus grand nombre? Comment? Alors que toutes les forces du système technico-économique, toutes les forces politiques rétrogrades et nationalistes militent dans le sens inverse. Billeter lui-même lève le doute en se demandant si la nécessaire « réflexion sur notre besoin et notre désir essentiels » sera " encore possible si les choses tournent mal? Si la catastrophe l'emporte et que commence la lutte de tous contre tous? $»^{21}$

C'est une catastrophe d'ordre politique et xénophobe que Billeter envisage. Mais, la catastrophe voire le cataclysme qui se profile de plus en plus est d'ordre climatique et sanitaire. Et il me semble que l'humanité tout entière - parce que comment limiter la solution à un seul continent-réagirait uniquement une fois précipitée dans la catastrophe. Dans un texte à la fois ludique et sérieux, car l'avenir de nos enfants et des leurs est une chose sérieuse, j'avais évoqué un avenir possible qui émergeait en réponse à des événements climatiques et sanitaires catastrophiques, des scénarios dans lesquels beaucoup de gens mourraient avant que les survivants ne reprennent les choses en main. 
Je cite ci-dessous un passage qui, depuis que je l'ai écrit il y a cinq ans me semble plus crédible et beaucoup moins farfelu :

Peu après la Chinatownisation de Shanghai, la Chine fut frappée par la peste du cochon volant, une confluence de deux sous-types du virus A-H1N1, l'un porcin, l'autre aviaire - des scientifiques chinois avaient croisé des canards et des cochons pour en produire du cochon laqué prêt à manger. Le résultat était une bête facilement sujette à ce nouveau virus, qui se transmettait facilement à l'homme. Une épidémie avait dévasté la mégapole de Shanghai, pour la réduire en véritable Zéropolis. Des dizaines de millions de Chinois y avaient péri, et également pas mal de Français qui s 'y étaient installés pour faire fortune. Plus de quartiers nobles, plus de quartiers tragiques...rien qu'une Old New Babylon.

Puis une fois les nouvelles limites fixées il n'y avait plus les ressources énergétiques pour alimenter cette ville spectaculaire. Et comme tout le monde le sait, étant donné que les Chinois ont tout inventé (la poudre, l'imprimerie, le papier, la glace, les pâtes), je dois vous dire que la psycho-géographie elle aussi fut inventée en Chine par un certain philosophe chinois taoïste qui s'appelle Zhuangzi 莊子, qui déjà deux millénaires avant Ellul et le mouvement de 2020 avait élaboré une pensée qui prônait les limites. Mais là où Ellul appréhendait que l'Homme eût évincé Dieu et mis à la place de la Création la Nature comme l'Autre de l'Homme devenu tout puissant, Zhuangzi ne voyait aucune séparation entre une autorité divine et l'Homme, ou entre l'Homme et «la Nature ». L'Homme faisait partie du processus de la vie et de la mort. Il n'exhortait pas l'Homme à vivre en harmonie avec la Nature, mais plutôt à prendre sa place parmi les myriades de choses animées et inanimées, à intégrer le processus de vie et de mort.

Après le GEL - Grand établissement de limites - le fait que les instituts Confucius établis à la fin du vingtième siècle furent fermés au profit des instituts Zhuangzi, dont la mission principale était de participer à l'enrichissement de la psychogéographie au service de l'imagination d'une nouvelle culture humaine, démontre bien que le rêve Taoïste avait fait son « chemin » (ce que Tao veut dire), et que finalement l'histoire a donné raison à Zhuangzi. N'avait-il pas dit il y a 2,400 ans :

Les idiots pensent qu'ils sont réveillés, ils sont si sûrs de savoir ce qu'ils sont, des princes, des bergers. Quelle sottise! Vous et Confucius, vous êtes tous les deux des rêves, et moi qui vous décris comme un rêve, je suis un rêve aussi. (Qi wu lun)

C'était un sentiment pareil que Calderón de la Barca évoqua quand il écrivit : « Toda la vida es sueño y sueños son [toute la vie est un songe et les songes mêmes ne sont que songes] $\gg .^{22}$

Malheureusement, je pense, et j'espère avoir tort, que de pareilles choses vont devoir se produire avant que les sociétés ne réagissent, car l'apparition de millions de réfugiés n'a guère d'impact moral sur les habitants des pays riches qui sont à la source des désastres humanitaires. Ce sera uniquement lorsque ceux qui détiennent le pouvoir et la richesse seront également atteints par de telles catastrophes, qu'une réaction sera possible. Nous savons déjà quelles sont les stratégies qu'il faudrait mettre en place, quels comportements il faudrait changer, la question demeure de savoir comment y arriver, par quelle force sociétale.

Mais la deuxième question que j'ai posée au départ et qui concerne la nécessité d'instituer un nouvel imaginaire, reste à élaborer. Il est une évidence, comme Ellul et Gorz l'ont démontré, que le travail productiviste est non seulement dépassé en tant qu'activité humaine, mais qu'il est indéniablement nuisible à notre environnement, et de plus, se fait au détriment de l'épanouissement humain. ${ }^{23}$ 

travail, et démontré les bénéfices d'une réduction massive du temps passé dans ce qu'il considérait être une activité destructrice de l'esprit humain, tout en nous rappelant l'importance du jeu dans la vie humaine. Il fustigeait la formule : « chi non lavora non mangi » (qui ne travaille pas, ne mange pas), car pour Rensi : « il rapporto sta tra il diritto di mangiare e quello di non lavorare », « il faudrait plutôt parler d'un droit au non-travail plutôt qu'un 'droit au travail' ». ${ }^{24}$

C'est aussi Rensi qui nous rappelle que le philosophe et poète Suisse Henri Frédéric Amiel (1821-1881) chantait déjà au dix-neuvième siècle, comme notre cher Baudelaire, les éloges de la flânerie et de la rêverie :

Nous sommes trop affairés, trop encombrés, trop occupés, trop actifs! Nous lisons trop! Il faut savoir être oisifs. Dans l'inaction attentive et recueillie, notre âme efface ses plis. La rêverie, comme la pluie des nuits, fait reverdir les idées fatiguées et pâlies par la chaleur du jour. En se jouant, elle accumule les matériaux pour l'avenir et les images pour le talent. La flânerie n'est pas seulement délicieuse ; elle est utile. C'est un bain de santé qui rend la vigueur et la souplesse à tout l'être ; c'est le signe et la fête de la liberté. ${ }^{25}$

Rensi, et Amiel avant lui, critiquait le travail pour son impact négatif sur l'être humain, sa manière d'aliéner l'individu de lui-même et de le séparer d'Autrui et du monde. Ils ne pouvaient pas encore apercevoir ce qu'Ellul verrait plus tard, c'est-à-dire l'impact destructeur du travail sur le monde physique et biologique. Au vingt-et-unième siècle, il existe par conséquent un double raisonnement pour réduire de manière drastique, sinon abolir, le travail: (i) la nécessité de sortir du productivisme qui, couplé avec la consommation de masse, est en train d'impacter de manière peut-être déjà irréparable l'existence humaine sur notre planète, et puis (ii) la mise en place d'un nouvel imaginaire qui libèrerait les forces créatrices et qui, primerait la créativité, un imaginaire dans lequel le jeu, la flânerie, la rêverie constitueraient non seulement des éléments, mais des instruments fondamentaux pour arriver à une nouvelle façon, non seulement de survivre, mais de vivre.

Mais, pour sortir du productivisme et de la consommation de masse il faudrait s'attaquer au travail, et par conséquent au capital. Evoquant l'universitaire canadien Moishe Postone, qui avait révélé dans ses recherches la différence entre valeur et richesse, Gorz souligne que «le capital, le travail, les deux sont indissociables, et si l'on veut se débarrasser du capital, il faut se débarrasser du travail. $»^{26}$ La logique du capital nuit également à la mise en place d'un imaginaire libérateur des forces et des espaces créateurs, car «la stratégie de domination du capital consiste à empêcher la naissance d'espaces d'autres activités et à conduire les gens à considérer que le travail rémunéré, le travail-marchandise, travail-emploi, est indispensable à leur dignité et à leur épanouissement personnel ».27

L'essentiel à présent est de penser à la manière de se servir du temps de travail une fois l'imaginaire du travail brisé, et les limites et les seuils appliqués; car une politique des limites doit nécessairement passer par une réduction massive du temps de travail.

Encore une fois, il ne suffit pas d'imposer et de s'imposer des limites, ce qui est déjà en train de se faire dans les pays les plus affluents de la planète. Cela serait un gaspillage total, qui en tout cas ne fonctionnerait pas longtemps, de simplement « libérer » les gens du travail pour qu'ils passent leur temps devant les écrans. André Gorz avait insisté sur ce point :

Transtext(e)s Transcultures 跨文本跨文化, 13 | 2018 
Gorz précisait qu'une politique de réduction du temps de travail devait forcément s'accompagner d'une véritable politique du "temps». Pour lui, en effet le temps libéré n'avait de sens que s'il pouvait servir à façonner librement les relations et la collaboration sociales. En 1997, Gorz ralliera à l'idée d'allocation universelle d'un revenu d'existence. ${ }^{28}$

Il y aurait la possibilité de créer de l'art public, de repenser et de construire de manière écologique notre "aménagement» du territoire de manière intentionnellement plus ludique, une sorte de « New Deal » post-consumériste, et post-travail. Il faudrait repenser nos systèmes de santé les rendant plus humains, et en finir avec les traitements et la dominance d'une médecine industrielle. Il faudrait replanter les plantes médicinales en provenance de la planète entière, partager les connaissances des médecines d'antan et d'ailleurs. De nouvelles technologies seraient développées uniquement là où un besoin communautaire serait clairement démontré et pas seulement parce nous serions en mesure de les développer. ${ }^{29} \mathrm{Il}$ faudrait construire de nouvelles habitations, restaurer les anciennes, tout cela en respectant les souhaits des communautés, et des peuples. Et dans chaque voisinage, la créativité artistique, les arts plastiques, l'art public, les spectacles théâtraux, la musique auraient une place centrale. Toutes ces activités seraient au cœur de l'enseignement avec l'histoire mondiale à la place d'honneur, une histoire qui prendrait en compte l'impact négatif du colonialisme européen et du capitalisme industriel.

Et puis l'urgence dans les trente ans à venir serait l'accueil des réfugiés. Il faudrait que les gens s'organisent pour gérer toutes les crises liées au changement climatique: en particulier les migrants et les réfugiés qui auraient besoin et continueraient à avoir besoin pendant longtemps d'aide pour reconstruire leurs vies. Il faudrait aussi beaucoup de personnes pour enseigner les langues; les barrières linguistiques devraient tomber, et la dominance de quelques langues serait remplacée par un nouveau multilinguisme. Ainsi, dans le petit nombre d'heures travaillées, il y aurait énormément à accomplir pour tous.

Comme Rensi nous l'a enseigné, il y a le travail en tant que contrainte, aliénant et répétitif, et puis il y a le travail-jeu, dans lequel on peut trouver du plaisir : «celui des enfants qui se poursuivent jusqu'au bout de leurs forces, des jeunes gens qui se donnent au sport jusqu'à épuisement, de l'artiste ou du penseur qui, sans relâche, compose des vers ou de la musique, peint, cherche, médite, écrit sur des questionnements scientifiques ou philosophiques $»^{30}$

41 Comme Gorz et d'autres nous l'ont montré, le travail contraignant mais nécessaire peut être organisé de manière hebdomadaire, dix heures par semaine par exemple, ou peutêtre davantage, quitte à accorder des années sabbatiques entières durant la vie des individus afin que les gens, jeunes ou vieux, puissent se consacrer à leurs passions à plein temps. Ainsi, une vie sans marchandises ne serait pas vécue comme une misère, comme une pénurie, mais plutôt comme une libération. Là déjà, nous voyons l'esquisse d'un programme non seulement pour assurer la survie mais pour commencer à construire une vie digne pour nos descendants.

Comment y arriver? On ne peut que revenir à Castoriadis, car s'il n'a pas élaboré à quoi ressemblerait une société après l'imposition des limites, il a tout à fait raison de souligner la nécessité de procéder de manière réellement démocratique, car la mise en place des limites est "la tâche et l'œuvre des individus (des citoyens) éduqués par et pour la démocratie ». ${ }^{31}$ En effet, à quoi bon "sauver la planète » si c'est pour vivre sous une 
dictature qui imposerait non seulement des limites de consommation mais également des limites idéologiques?

Mais cette éducation dont parle Castoriadis doit également être une exigence populaire, et cette éducation révolutionnaire doit déjà être gérée de manière démocratique comme un vaste réseau d'universités populaires et non pas par l'état. Les mouvements populaires d'aujourd'hui laissent espérer que la troisième décennie du vingt-et-unième siècle sera un moment ou l'humanité se réveillera enfin de son long sommeil.

\section{NOTES}

1. Quentin Hardy, introduction à Bernard Charbonneau et Jacques Ellul, Nous sommes des révolutionnaires malgré nous, Paris, Seuil, 2014, p. 36.

2. Cornelius Castoriadis, Fait et à faire: Les carrefours du labyrinthe 5, Paris: Seuil, 1997, p. 220.

3. Quentin Hardy in Charbonneau et Ellul, p. 36.

4. Jacques Ellul, Le Système technicien, Paris, Calman-Lévy, 1977, Le Cherche midi 2012 p. 305.

5. Ellul, Système technicien, p. 305 n. 25.

6. Castoriadis, Labyrinthe, V, p. 207.

7. Castoriadis, Écrits politiques IV : Quelle Démocratie tome 2, Paris, Éditions du Sandre, 1990, p 384.

8. Castoriadis, Écrits politiques, IV, p. 387

9. Voir Gregory B. Lee, China Imagined: From European Fantasy to Spectacular Power, London, Hurst \& Co., 2018, passim.

10. Confucius, nom latinisé de Kǒng Fūzǐ 孔夫子, « maître Kong ».

11. Le poème est daté 1804, imprimé en 1808. Les paroles sont familières aux anglais par le biais de l'hymne « Jerusalem » (1916), musique de Sir Hubert Parry.

12. Jean François Billeter, Études sur Tchouang-tseu p. 48.

13. Guo Xiang est décédé en 312.

14. Billeter, Études, p. 48. Billeter emploie le système de transcription phonétique de l'École française d'Extrême-Orient (EFEO), conçu par le jésuite français Séraphin Couvreur;

15. Billeter Études, p. 60

16. Billeter Études, p. 61

17. Ellul, Exégèse des nouveaux lieux communs, 1966, p. 17.

18. Ellul, Exégèse p. 18 
19. Gregory B. Lee, «Le cadeau empoisonné de Versailles ou la Chine à la manivelle de l'orgue de barbarie », Mouvements, vol. 72, no. 4, 2012, pp. 79-88. https://doi.org/10.3917/ mouv.072.0079

20. Billeter, Demain l'Europe, Paris, Allia, 2019, p. 37.

21. Billeter, Demain l'Europe, p. 38.

22. Gregory B. Lee in Nathalie Caritoux et Florent Villard, Nouvelles Psychogéographies, Paris, Mimésis, 2016.

23. Voir Gregory B. Lee, "The Commonplace of "Work as Freedom" and why the Left is so attached to its virtues ", Blog: La longue marche de Gregory Lee, Mediapart, 2 mars 2017, https://blogs.mediapart.fr/gblee/blog/120317/commonplace-work-freedom-and-whyleft-so-attached-its-virtues

24. L'Irrazionale, Il Lavoro, L'Amore, Milan, Unitas, 1923, p. 252-253 ; Contre le Travail, traduction de Marie-José Tramuta, précédée par Gianfranco Sanguinetti, «L'Audace de Giuseppe Rensi », Paris, Allia, 2017, p. 72.

25. Fragments d'un journal intime, Genève, George \& Co, tome I, p. 52.

26. André Gorz, Penser l'Avenir: Entretien avec François Noudelmann, Paris, La Découverte, 2019, pp. 53-54.

Voir aussi, Moishe Postione, «The Current Crisis and the Anachronism of Value: A Marxian Reading » in Continental Thought \& Theory: A Journal of Intellectual Freedom, Vol. 1, 4: 150 Years of Capital, pp. 38-54.

27. Gorz, Penser l'Avenir, p. 60.

28. Christophe Fourriel, Préface à André Gorz, Penser l'Avenir, p. 10.

29. Comme dit Billeter, Demain l'Europe p. 36, «La recherche n'obéira plus à la loi de l'infini capitaliste ».

30. Rensi, Contre le Travail, p. 53.

31. Castoriadis, Labyrinthe, V, p. 207.

\section{RÉSUMÉS}

À la lumière d'une prise de conscience tardive de la crise climatique, le besoin de limiter - la production, la consommation, la domination de la «Nature » et l'exercice du pouvoir - est devenu urgent. Cela étant, les limites seules suffiront-elles à infléchir les plus néfastes des répercussions, et même si elles y suffisaient, par le biais de quel mécanisme politique y arriverait-on? Cet article démontre comment le double mouvement du capitalisme et de la techno-science (Ellul, Charbonneau, Castoriadis) a constamment mené à un stade de l'histoire de l'humanité où la limitation intervient trop tard pour sauver le système existant. La société mondiale tout entière doit dorénavant modifier de manière radicale son exploitation des ressources planétaires pour créer une nouvelle culture dans laquelle la production et le travail inutile (Rensi, Gorz) seront remplacés par une façon de vivre qui respectera non seulement l'environnement où habite l'humanité, mais l'humanité elle-même. Pour arriver à une telle transformation culturelle, l'exercice même du pouvoir doit être bridé (Castoriadis, Zhuangzi, Billeter) pour céder la place à une pratique démocratique et populaire. 
The need for the implementation of limits - to production, to consumption, to our domination of 'Nature', to the wielding of power - has, with humanity's belated collective awareness of its planet's climate crisis, become urgent. But are limits alone sufficient to change the more disastrous possible outcomes, and even if they were by what political mechanism can they be brought about? This article shows how the dual movement of capitalism and unbridled technoscience (Ellul, Charbonneau, Castoriadis) has unrelentingly propelled humanity towards a stage in its history where limitation is already too late to save the system as we have known it. Global society must now fundamentally alter its use of the planet's ressources and create a new culture in which production and futile labour (Rensi, Gorz) give way to a way of life that not only respects humanity's environment but humans themselves. To achieve such a cultural shift, power itself must be bridled (Castoriadis, Zhuangzi, Billeter) and a new popular, democratic practice put in place.

\section{AUTEUR}

\section{GREGORY B. LEE}

Gregory B. Lee is Professor of Chinese and Transcultural Studies at the University of Lyon 3. He is a graduate of the School of Oriental and African Studies, London, and of Peking University. He previously taught at the universities of Cambridge, London, Chicago and Hong Kong. His research interests include cultural and intellectual history, Chineseness, the postcolonial, 'minor' and 'marginal' cultures, diasporic memory and the impact of globalization on all of these. His most recent book is China Imagined : From European Fantasy to Spectacular Power, London, Hurst \& Co., 2018. 